NOTE TECHNIQUE

\title{
Influence de la teneur en matière grasse du lait sur la précision des dosages de lactose avec l'appareil IRMA
}

\author{
par \\ R. GRAPPIN et J.C. COLLIN \\ I.N.R.A., Station Expérimentale Laitière - 39800 Poligny
}

\section{INTRODUCTION}

Actuellement, la détermination de la teneur en lactose du lait peut être faite très rapidement à l'aide de méthodes automatiques modernes et notamment avec l'appareil IRMA (Adda et al., 1968). Dans la plupart des pays, cet appareil est utilisé essentiellement pour doser la matière grasse et les protéines du lait ; le dosage du lactose n'est effectué que dans les pays intéressés par la détermination (le calcul) de l'extrait sec du lait.

Comme le dosage du lactose est effectué avec l'IRMA, pratiquement sans coût supplémentaire, Renner (1972) a étudié la possibilité de l'utiliser comme test permettant de détecter les laits de mammite. On sait en effet que le taux de lactose décroît lorsque la vache est atteinte de mammite, la baisse étant proportionnelle à la gravité de l'infection (Tolle et al., 1971). Mais cette baisse est relativement faible, aussi, est-il important de disposer d'une méthode qui soit précise et notamment sensible. Les études concernant l'appareil IRMA donnent pour le lactose un écart-type de précision de l'ordre de 0,6 à $0,7 \mathrm{~g} / \mathrm{kg}$. Cette précision peut-elle être améliorée afin d'augmenter la sensibilité des mesures ?

Goulden (1964) a montré qu'à chacune des longueurs d'onde utilisées pour la détermination des constituants du lait, il existe toujours une interaction de la part des autres constituants. C'est ainsi qu'à $9,6 \mu \mathrm{m}$, longueur d'onde à laquelle se fait le dosage du lactose, le taux de matière grasse et le taux de protéines du lait influencent la mesure faite au moyen de l'appareil. D'après la varia- 
bilité des teneurs en matière grasse et en protéines du lait et les coefficients d'extinction propres à chaque élément, Goulden a estimé que l'erreur provenant du taux de matière grasse pouvait être au maximum de \pm 5 p. 100 de sa valeur moyenne et celle du taux de protéines de $\pm 1,5$ p. 100 .

Compte tenu d'une part, de la corrélation existant entre les teneurs en matière grasse et en protéines, et d'autre part, du fait que la matière grasse entraîne une erreur par défaut et les protéines une erreur par excès, le constructeur d'IRMA a estimé inutile toute correction des résultats du lactose en fonction des autres composants. Toutefois, ayant observé au cours des précédentes expérimentations, que le taux de matière grasse du lait influençait notablement les résultats des dosages du lactose, nous avons entrepris de déterminer expérimentalement, sur des laits individuels, l'influence exacte de la richesse en matière grasse sur la précision des dosages du lactose.

\section{MATERIEL ET METHODES}

\section{1) Nature des échantillons de lait}

L'essai porte sur 60 échantillons de laits individuels provenant de vaches à tous les stades de lactation, réparties dans 4 étables du Jura. Le taux de matière grasse des laits varie entre 22 et $62 \mathrm{~g} / \mathrm{kg}$, celui des matières azotées ( $\mathrm{N}$ total x 6,38) entre 28 et $49 \mathrm{~g} / \mathrm{kg}$ et le taux de lactose varie entre 42 et $51 \mathrm{~g} / \mathrm{kg}$.

\section{2) Détermination des teneurs en matière grasse, protéines, lactose}

\subsection{Au moyen de l'appareil IRMA}

Les teneurs en matière grasse, protéines et lactose ont été déterminées au moyen de l'appareil IRMA.

Tous les dosages ont été faits en triple sur chacun des échantillons additionnés de 0,1 p. 100 de $\mathrm{K}_{2} \mathrm{Cr}_{2} \mathrm{O}_{7}$ et conservés au plus $8 \mathrm{j}$ à $4^{\circ} \mathrm{C}$. Préalablement, l'appareil IRMA avait été étalonné à l'aide des solutions normalisées préconisées par le constructeur.

L'écart-type de répétabilité des dosages de lactose avec l'IRMA est de $0,20 \mathrm{~g} / \mathrm{kg}$.

\subsection{Par dosage chimique}

La teneur en lactose a été déterminée au moyen de la méthode officielle française Potterat-Eschmann (A.M. 8 janvier 1970). Tous les dosages ont été faits en double sur des laits conservés au congélateur à $-30^{\circ} \mathrm{C}$. 


\section{RESULTATS ET DISCUSSION}

\section{1) Influence des différents composants du lait sur les dosages du lactose}

A $9,6 \mu \mathrm{m}$, les mesures effectuées par l'appareil IRMA peuvent être exprimées en fonction de la concentration du lait en lactose d'une part, mais également en fonction des teneurs en matière grasse et en protéines d'autre part, soit :

Lecture IRMA $=\mathrm{a}+\mathrm{b}_{1} \times$ lactose chimique $+\mathrm{b}_{2} \times$ matière grasse $+\mathrm{b}_{3} \times$ protéines.

Les résultats obtenus sont les suivants : $b_{1}=1,000 \pm 0,051$.

$$
\begin{aligned}
& b_{2}=-0,084 \pm 0,016 . \\
& b_{3}=0,063 \pm 0,032 .
\end{aligned}
$$

Dans le lait, c'est l'eau qui absorbe une grande quantité d'énergie infra-rouge et ceci pratiquement quelle que soit la longueur d'onde, tandis que pour les différents composants, l'absorption se fait essentiellement de manière sélective.

Par conséquent, pour une longueur d'onde donnée, soit, par exemple, 9,6 $\mu \mathrm{m}$ pour le dosage du lactose, toute variation de la concentration en matière grasse et en protéines, entraîne une variation équivalente (en volume) de la teneur en eau et se traduit par une absorption d'énergie infra-rouge qui est inversement proportionnelle à la teneur en matière grasse et en matières azotées. Ainsi s'explique le signe négatif du coefficient $b_{2}$ de la matière grasse. L'influence positive du taux de protéines indique que l'énergie infra-rouge absorbée par les protéines à $9,6 \mu \mathrm{m}$ est plus importante que celle de l'eau. Les valeurs de ces coefficients sont en accord avec celles qui ont été déterminées en utilisant d'autres méthodes par Goulden $\left(b_{2}=-0,996 ; b_{3}=0,054\right)$ et par Biggs $\left(b_{2}=-0,081\right.$; $\left.\mathrm{b}_{3}=0,054\right)$.

En tenant compte des teneurs en lactose, matière grasse et matière azotée, l'écart-type résiduel des lectures IRMA par rapport aux trois variables est de $0,36 \mathrm{~g} / \mathrm{kg}$.

\section{2) Correction en fonction du taux de matière grasse}

$\mathrm{Si}$ on porte sur un graphique (fig. 1) les écarts entre les lectures IRMA et les valeurs obtenues par voie chimique, en fonction du taux de matière grasse des laits, on constate une corrélation négative significative $(\mathrm{r}=-0,78)$ indiquant que $61 \mathrm{p} .100$ environ des différences sont dues aux variations du taux butyreux des laits. La pente de la droite est de $-0,07$. Ce coefficient diffère légèrement de la valeur $b_{2}$ calculée plus haut en raison de l'influence des protéines qui se trouve confondue avec celle de la matière grasse. 


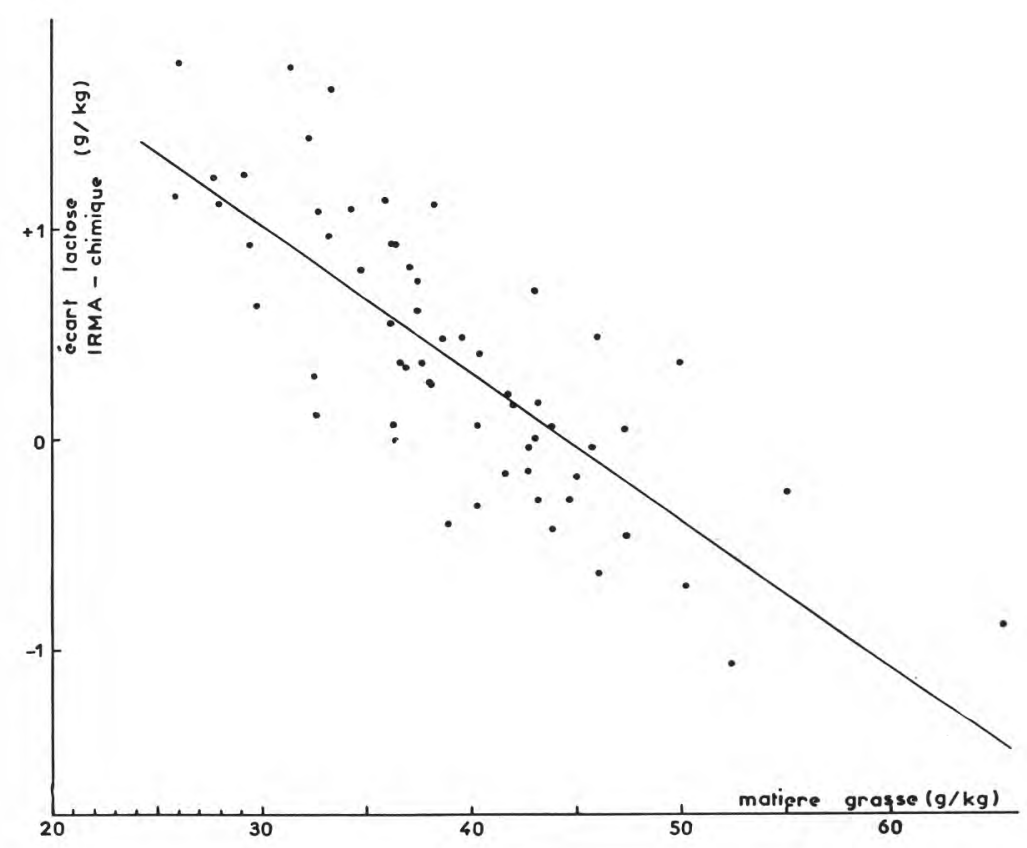

fig. 1

Relation entre la teneur en matière grasse du lait et les différences observées entre les dosages de lactose effectués par une méthode chimique de référence et par l'appareil IRMA ( $b=-0,07 ; \mathrm{r}=-0,78)$.

Dans le cas d'un dosage normal du lactose, la précision de l'IRMA exprimée par l'écart-type résiduel est de $0,64 \mathrm{~g} / \mathrm{kg}$ avec un coefficient de corrélation de 0,957 (fig. 2) ; en corrigeant les lectures IRMA en fonction du taux de matière grasse du lait, avec un facteur de correction de $0,07 \mathrm{~g}$ par gramme de matière grasse, on obtient un écart-type de 0,41 g/kg et un coefficient de corrélation de 0,980 (fig. 3).

Cette précision est bonne, elle est proche de celle qui a été obtenue avec l'équation de régression multiple $\left(\sigma_{\mathrm{r}}=0,36 \mathrm{~g} / \mathrm{kg}\right)$.

L'appareil étant déjà muni d'un système de réglage permettant de corriger le taux de protéines en fonction du taux de matière grasse, il serait possible, sans grande modification, d'effectuer une autre correction avec un facteur différent ( $-0,07$ au lieu de $-0,21)$ au niveau du taux de lactose. Cette correction améliorerait, dans le cas des laits individuels, la précision des dosages de lactose de 36 p. 100 environ. 


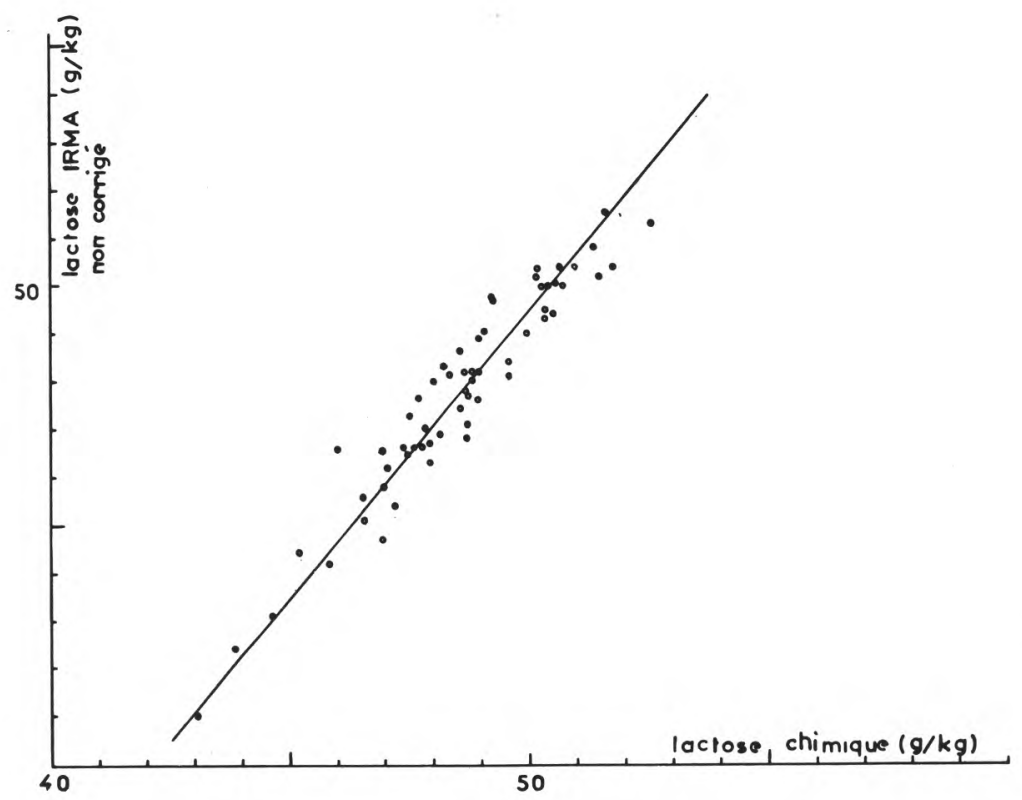

fig. 2

Relation entre les dosages de lactose effectués par une méthode chimique de référence et par l'appareil IRMA sans correction en fonction du taux de matière grasse $(\sigma=0,64 ; \mathrm{r}=0,957)$.

\section{Ré s u m é}

Une expérience portant sur 60 échantillons de laits individuels a montré que la précision des dosages de lactose effectués par l'appareil IRMA était influencée de manière sensible par la teneur en matière grasse des laits. En effectuant une correction des résultats lactose IRMA de $-0,07 \mathrm{~g} / \mathrm{kg}$ par gramme de variation du taux de matière grasse des laits, la précision des dosages passe de $0,64 \mathrm{~g} / \mathrm{kg}$ à $0,41 \mathrm{~g} / \mathrm{kg}$.

Cette correction devrait pouvoir être faite automatiquement par l'appareil. 


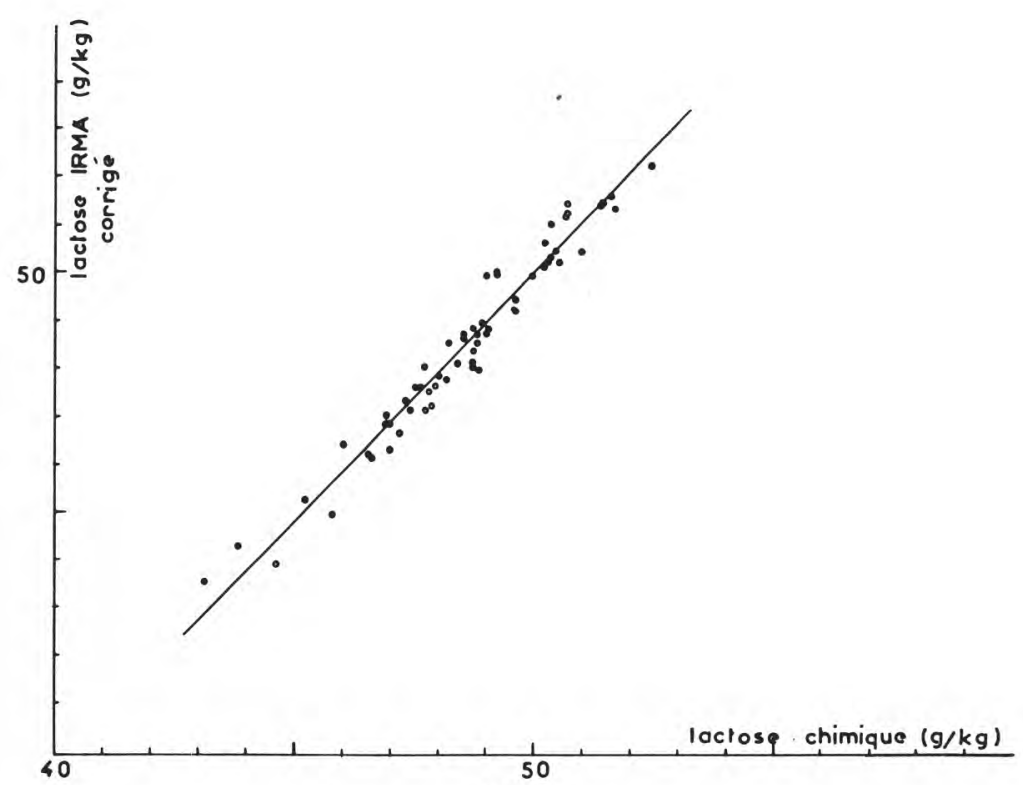

fig. 3

Relation entre les dosages de lactose effectués par une méthode chimique de référence et par l'appareil IRMA après correction en fonction du taux de matière grasse $(\sigma=0,41 ; \mathrm{r}=0,980)$.

\section{S u m m a r y}

A test carried out on 60 individual milk samples has shown that the accuracy of the lactose determination with the Infra-RedMilk-Analyser (IRMA) is mainly influenced by the fat content of milk. Using a correction of 0.007 p. 100 of lactose readings for 0.1 p. 100 change in fat content, the s.d. of the accuracy changes from 0.064 p. 100 to 0.041 p. 100 .

This correction should be done automatically by the instrument.

Reçu pour publication le 25 juin 1974.

\section{Bibliographie}

Goulden (J. D. S.) (1964). - Analysis of milk by infra-red absorption. J. Dairy Res., 31, 273-284.

Adda (J.), Blanc-Patin (E.), Jeunet (R.), Grappin (R.), Mocouot (G.), Poujardieu, RicoRDEAU (G.) (1968). - Essais d'utilisation de l'Infra-Red-Milk-Analyser. Le Lait, 145-154 et 273-302.

RENneR (E.). - Möglichkeit der Feststellung von Entererkrankungen mit dem Infrarot-Milchanalysator (IRMA). Deutsche Molkerei-Zeitung, 93, 71-77.

Tolle (A.), Heeschen (W.), Reichmuth (J.), Zeidler (H.) (1971). - Counting of somatic cells in milk and possibilities of automation. Dairy Sc. Abstr., 33, 875-879. 